\title{
Visualization of deep choroidal vasculatures and measurement of choroidal vascular density: a swept-source optical coherence tomography angiography approach
}

Erqian Wang ${ }^{1,2}$, Xinyu Zhao ${ }^{1,2}$, Jingyuan Yang ${ }^{1,2}$ and Youxin Chen ${ }^{1,2^{*}}$ (D)

\begin{abstract}
Background: To investigate swept-source optical coherence tomography angiography (SS-OCTA) for deep choroid visualization and choroidal vascular density (CVD) measurement.

Methods: Healthy subjects and central serous chorioretinopathy (CSC) patients were recruited for macular SS-OCTA scans. We evaluated OCTA images at various depths to determine an optimal depth for visualizing choroidal vasculatures. We measured CVD with binarized OCTA images at the optimal depth. In healthy subjects, CVD was studied for its correlation with age, axial length (AL), and subfoveal choroidal thickness (SCT). In CSC eyes, CVD was compared with matched controls.

Results: Ninety-one healthy eyes and 22 CSC eyes were included. SS-OCTA could display deep choroidal vasculatures as dark signals, with $100 \mu \mathrm{m}$ beneath BM as the optimal depth. In healthy subjects, the CVD at $100 \mu \mathrm{m}$ beneath BM $(56.5 \pm 10.9 \%)$ was significantly correlated with SCT $(P=0.004)$ but not with age $(P=0.49)$ or $\mathrm{AL}(P=$ 0.72). In CSC eyes, the CVD at $100 \mu \mathrm{m}$ beneath BM (62.3 $\pm 6.6 \%)$ was larger than that in 22 matched controls (54.1 \pm 8.0\%) $(P=0.001)$. The difference remained statistically significant after adjusting for SCT $(P=0.02)$.

Conclusions: SS-OCTA can be used for visualizing deep choroidal vasculatures. CVD measured by OCTA at $100 \mu \mathrm{m}$ beneath BM is a useful parameter for quantifying choroidal vascular status.
\end{abstract}

Keywords: Optical coherence tomography angiography, Swept-source, Choroidal vascular density, Subfoveal choroidal thickness, Central serous chorioretinopathy

\footnotetext{
* Correspondence: chenyouxinpumch@hotmail.com; chenyx@pumch.cn

${ }^{1}$ Key Laboratory of Ocular Fundus Disease, Chinese Academy of Medical Sciences, Beijing 100730, China

2Department of Ophthalmology, Peking Union Medical College Hospital,

No.1 Dongshuaifuyuan, Dongcheng District, Beijing 100730, China
}

C The Author(s). 2020 Open Access This article is licensed under a Creative Commons Attribution 4.0 International License, which permits use, sharing, adaptation, distribution and reproduction in any medium or format, as long as you give appropriate credit to the original author(s) and the source, provide a link to the Creative Commons licence, and indicate if changes were made. The images or other third party material in this article are included in the article's Creative Commons licence, unless indicated otherwise in a credit line to the material. If material is not included in the article's Creative Commons licence and your intended use is not permitted by statutory regulation or exceeds the permitted use, you will need to obtain permission directly from the copyright holder. To view a copy of this licence, visit http://creativecommons.org/licenses/by/4.0/. The Creative Commons Public Domain Dedication waiver (http://creativecommons.org/publicdomain/zero/1.0/) applies to the data made available in this article, unless otherwise stated in a credit line to the data. 


\section{Background}

Choroid, a layer of vascular tissue, plays an important role in physiological and pathological process of human eyes. Visualization of choroidal vasculature is helpful in improving our understanding in a number of retinal and choroidal diseases. The swept-source optical coherence tomography (SSOCT) technique permits deep choroid penetration. En-face OCT images of the choroid are useful for intuitive visualization of choroidal vasculatures and quantitative assessment of choroidal vascular density (CVD) [1, 2]. Quantification of CVD in deep choroid by en-face SS-OCT have been reported in healthy eyes [3], diabetic retinopathy [4], age related macular degeneration, polypoidal choroidal vasculopathy, and central serous chorioretinopathy (CSC) [5].

Optical coherence tomography angiography (OCTA), widely used for non-invasive imaging of retinal and choriocapillary microvasculatures $[6,7]$, has long been doubted for its value in deep choroid imaging. According to a previous report by Diaz et al. [8], intact retinal pigment epithelium (RPE) was regarded as a barrier for reliable detection of choroidal blood flow with OCTA. One previous study employed RTVue OCTA device for the quantification of deep choroidal vasculatures [9]. However, they used a built-in algorithm which was normally used for measuring choriocapillary flow density and was highly unlikely to quantify deep vasculatures correctly. Choi et al. [10] described a prototype SS-OCTA device at $1060 \mathrm{~nm}$ wavelengths with a $400 \mathrm{kHz}$ A-scan rate in imaging Sattler's layer of choroid. However, their equipment was commercially unavailable for wide application. Wang et al. [11] explored the ability of a commercially available SS-OCTA device to visualize and quantify the inner, middle, and outer choroidal vasculatures in a small series of eyes. However, there are still several gaps in current literature. First, the optimal depth for CVD measurements with SS-OCTA has not been identified. Second, the CVD measured with SS-OCTA has not been analyzed for its correlation with demographic factors. Third, the CVD measured with SS-OCTA has not been applicated in chorioretinal diseases.

To fill these gaps, we evaluated OCTA images at various choroidal depths with en-face OCT as reference standard, determined an optimal depth for visualizing choroidal vasculatures with OCTA, measured CVD with binarized OCTA image at the optimal depth, studied the OCTA derived CVD for its correlation with demographic factors in healthy subjects, and tested its usefulness in a series of CSC eyes. The purpose of this study is to improve our understanding in SS-OCTA for its capability in displaying deep choroidal vasculatures and quantifying deep choroidal vascularity.

\section{Methods}

Ethics committee of Peking Union Medical College Hospital approved this observational case series study. All participants gave informed consents, and we conducted the study in accordance with the ethical tenets of the Declaration of Helsinki.

\section{Subjects}

Healthy volunteers between 18 and 70 years without known systematic or ocular diseases were recruited and screened with a series of ophthalmic examinations including bestcorrected visual acuity (BCVA), intraocular eye pressure (IOP), slit-lamp biomicroscope, indirect ophthalmoscope, axial length (AL), and macular $12.0 \mathrm{~mm}$ radial SS-OCT scan (DRI OCT Triton plus, Topcon Corporation, Tokyo, Japan). Subfoveal choroidal thickness (SCT) was measured at the center fovea between outer edge of retinal pigment epithelium and choroidal-scleral interface. Subjects were excluded if they had any one of the following circumstances in one or both eyes: 1. BCVA of less than 20/20; 2. IOP of more than $21 \mathrm{mmHg}$ or less than $10 \mathrm{mmHg}$; 3. suspected vitreoretinal, choroidal or optic nerve diseases detected by screening examinations; 4 . media opacity resulting in poor-quality image; and 5. AL of more than $26.50 \mathrm{~mm}$ or less than $20.50 \mathrm{~mm}$.

A prospective consecutive series of eyes with acute or chronic CSC were recruited as one test group. Acute CSC was defined as a recent ( $<3$ months) onset of subretinal fluid through a focal leakage without prior episodes. Chronic CSC was defined as prolonged (> 3 months) onset of symptoms with widespread pigment epithelium abnormalities, with or without subretinal fluid. We measured AL and SCT for all CSC eyes. A group of age-, gender-, and AL- matched healthy controls were selected from the above eligible healthy subjects.

\section{OCTA scans and image acquisitions}

A $3.0 \times 3.0 \mathrm{~mm}$ macular SS-OCTA scan (DRI OCT Triton plus, Topcon Corporation, Tokyo, Japan) were performed in both eyes of all eligible healthy volunteers and in all CSC eyes. The OCTA device worked at $1050 \mathrm{~nm}$ wavelength with a $100 \mathrm{kHz}$ A-scan rate and a repeated scan count of 4 times. Each OCTA scan was comprised of $320 \times 320$ A-scans with an axial resolution of $8 \mu \mathrm{m}$. The built-in Topcon software allowed simultaneous construction of $300 \times 300$-pixel depth-resolved OCTA and en-face OCT images based on one single scan. Therefore, the OCTA and en-face OCT images were generated from exactly the same area and there was no need for manual registration. OCTA and en-face OCT image pairs were built at every $2.6 \mu \mathrm{m}$ beneath between Bruch membrane (BM) by designating the same value for the starting and ending depth of each single-pixel slab (Fig. 1) [12]. The program automatically gave a score evaluating the sharpness and contrast of image series. Eyes with image score < 50 were excluded. Two investigators independently evaluated image artefacts for all OCTA image series. The investigators also confirmed that the $\mathrm{BM}$ of all subjects 


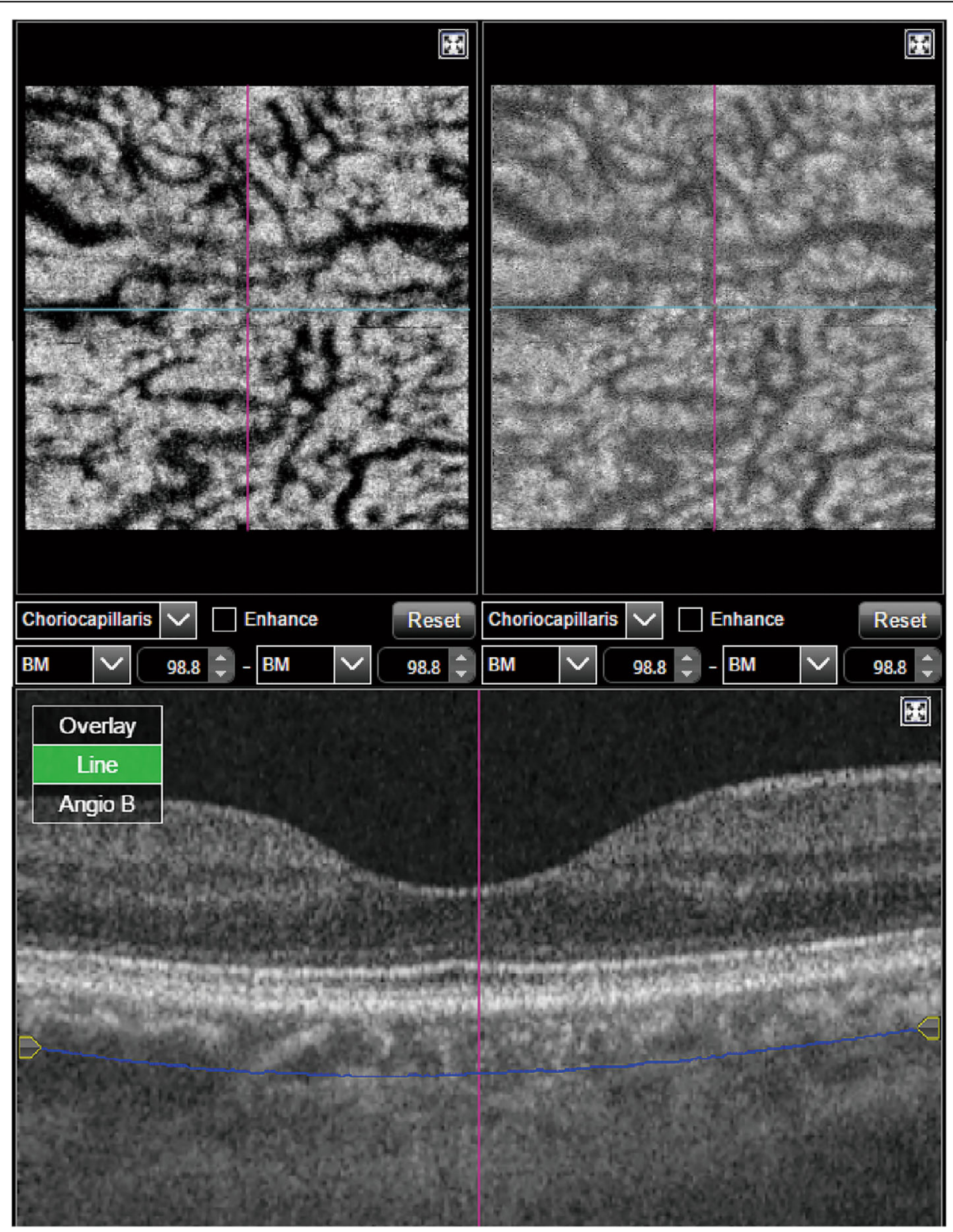

Fig. 1 Simultaneous construction of OCTA and en-face OCT image pairs based on one single OCTA scan. (Top left) The OCTA image of a singlepixel slab at $98.8 \mu \mathrm{m}$ beneath Bruch membrane (BM). (Top right) The en-face OCT image of the same slab. The OCTA and en-face OCT images showed similar pattern, in which dark stripes corresponded to the choroidal vessels. (Bottom) The position of the slab is shown by the arc line parallel to the BM

were recognized appropriately and there was no false segmentation due to pigment epithelium detachment or pachychoroid. Finally, only eyes without banding, blink, vessel doubling, stretching, out of window, crisscross, or $\geq$ 2 motion artefacts which were wider than primary branch vein diameter were included for further analysis $[13,14]$.

\section{Evaluation of Choroidal vasculature visualization at} various depths and determination of the optimal depth For healthy subjects, we collected choroidal OCTA and en-face OCT image pairs in two depth series, the fixed depth series with $50 \mu \mathrm{m}$ intervals and the percentile depth series with $12.5 \%$ SCT intervals. In fixed depth series, because the axial resolution of OCTA scan was $2.6 \mu \mathrm{m}$, the actual depths for image collection were $49.4 \mu \mathrm{m}, \quad 98.8 \mu \mathrm{m}, \quad 150.8 \mu \mathrm{m}, \quad 200.2 \mu \mathrm{m}$, $249.6 \mu \mathrm{m}, 299.0 \mu \mathrm{m}, 351.0 \mu \mathrm{m}, 400.4 \mu \mathrm{m}, 449.8 \mu \mathrm{m}$, $499.2 \mu \mathrm{m}$, and $551.2 \mu \mathrm{m}$ beneath BM. In percentile depth series, the actual depths for image collection in each eye were set as close to $12.5,25.0,37.5,50.0$, $62.5,75.0$, and $87.5 \%$ of its SCT beneath BM as possible. 
We evaluated healthy subjects for OCTA images at all selected depths with en-face OCT images as reference standard. First, three authors (E.W., X.Z., and J.Y.) independently assessed original OCTA images, resolved disagreement if there were any, and calculated the proportion of eyes with appropriate choroidal vessel display at each depth. Second, we binarized OCTA and en-face OCT image pairs and calculated the similarity between a binarized OCTA image and its en-face OCT counterpart, which was defined as the percentage of matched pixels in the total number of pixels compared. Image binarization was done with Otsu's method which calculated the optimum threshold for image binarization by minimizing intra-class variance [15] using ImageJ software by Wayne Rasband (National Institutes of Health, Bethesda, Maryland, USA; available at http://rsb. info.nih.gov/ij/index.html). Image comparison and similarity calculation were made with an algorithm developed by MATLAB software (MathWorks, Inc., Natick, MA, USA). Based on the above qualitative assessment and quantitative comparison, we determined an optimal depth, where OCTA images were most likely to display choroidal vessels appropriately and were most similar to its en-face OCT counterparts.

\section{Measurement of Choroidal vascular density at the optimal depth}

We measured CVD of healthy and CSC eyes with binarized OCTA image at the optimal depth. An OCTA image at the optimal depth was binarized with Otsu's method [15]. The black and white regions in binarized OCTA images were considered as vessel lumen and choroidal stroma, respectively. The CVD was defined as the proportion of black pixels from the total number of pixels in a binarized choroidal OCTA image [12] and was calculated using ImageJ software (Fig. 2).

\section{Statistical analysis}

The statistical analysis was performed with SPSS software version 22.0 (IBM, Inc., Chicago, IL, USA). Kolmogrov-Smirnov method was used for normality test. Univariate Pearson correlation analysis and multivariate linear regression were performed to study the correlation between CVD and potential correlating factors including age, AL, and SCT. One-way analysis of variance (ANOVA) was used for the comparison of age, axial length, SCT, and CVD between CSC eyes and matched controls. Analysis of covariance (ANCOVA) was also used for the comparison of CVD between CSC eyes and matched controls, with SCT adjusted as covariates. A $P$ value of less than 0.05 was considered statistically significant.

\section{Results}

We included 116 eyes of 58 healthy participants for SSOCTA scans. After excluding 2 eyes with blink artefact, 3 eyes with banding artefacts, 6 eyes with low image quality, and 14 eyes with at least two motion artefacts wider than primary branch vein diameter, we included 91 eyes from 51 healthy subjects (16 males and 35 females) for analysis. We also included 22 eyes with CSC (14 males and 8 females). All of the 5 acute CSC eyes and 13 of the 17 chronic CSC eyes had subretinal fluid overlying the scanned area. None of the CSC eyes had pachychoroid neovasculopathy. The characteristics of all included healthy eyes, CSC eyes and matched controls are shown in Table 1.

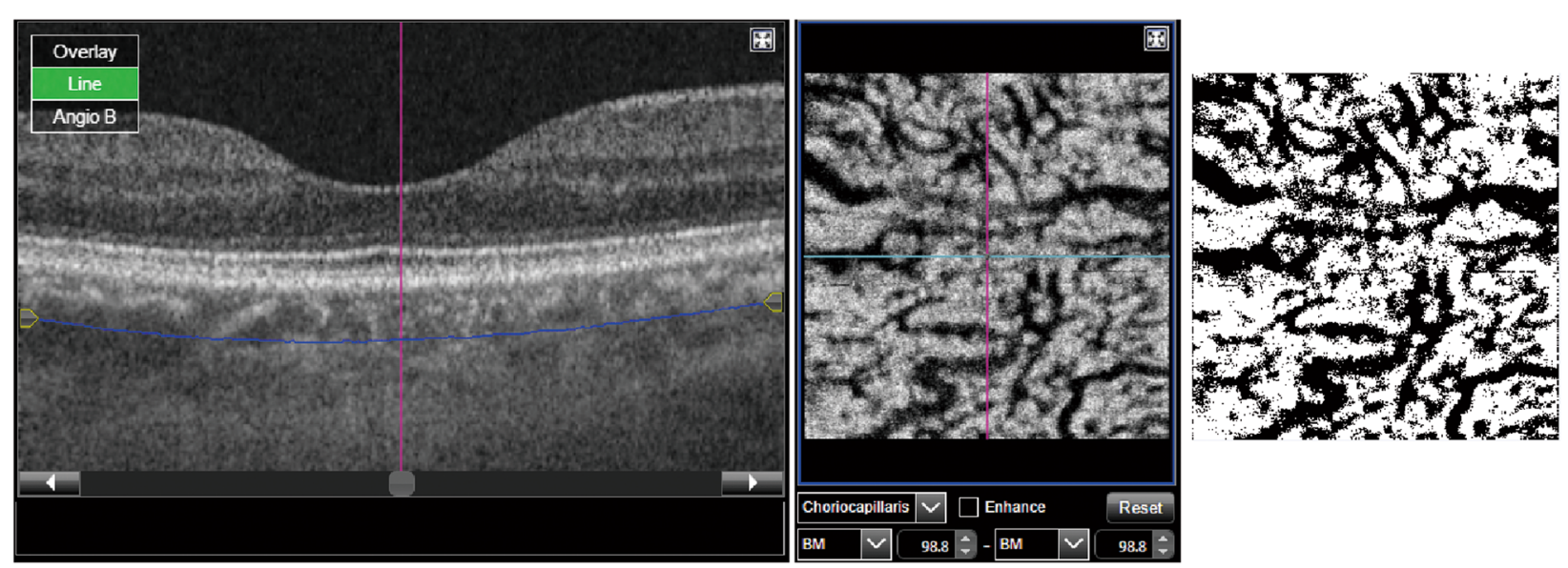

Fig. 2 Measuring choroidal vascular density with OCTA. (Left) B-scan of the macular area. (Middle) Construction of a single-slab OCTA image at $98.8 \mu \mathrm{m}$ beneath Bruch membrane. (Right) Binarization of the OCTA image. Choroidal vascular density was measured by the percentage of black pixels in the binarized image 
Table 1 Baseline characteristics of all included healthy eyes, CSC eyes, and matched controls

\begin{tabular}{lllll}
\hline Characteristics & Healthy eyes $(n=91)$ & CSC eyes $(n=22)$ & Matched controls ${ }^{*}(n=22)$ & $P^{\dagger}$ \\
\hline Age, years $($ mean \pm SD) & $39.4 \pm 12.3$ & $43.0 \pm 8.4$ & $42.9 \pm 11.9$ & 0.98 \\
AL, $\mathrm{mm}($ mean \pm SD) & $23.70 \pm 1.18$ & $22.82 \pm 1.08$ & $22.93 \pm 0.98$ & 0.70 \\
$\mathrm{SCT}, \mu \mathrm{m}$ (mean $\pm \mathrm{SD})$ & $333.2 \pm 94.2$ & $547.3 \pm 118.3$ & $374.1 \pm 101.7$ & $<\mathbf{0 . 0 0 1}$
\end{tabular}

Statistically significant $P$ value is shown in bold

* Matched controls were selected from the included healthy eyes

$+P$ values are from one-way analysis of variance (ANOVA) between CSC eyes and matched controls

CSC central serous chorioretinopathy, AL axial length, SCT subfoveal choroidal thickness, SD standard deviation

Patterns of Choroidal OCTA images at various depths and the optimal depth for Choroidal vasculatures visualization OCTA images at choriocapillaris exhibited granular pattern. As imaging plane went deep, OCTA images displayed choroidal vessels as dark stripes, which were similar to en-face OCT images. OCTA images at extremely deep level of choroid turned blurry or even black due to significant signal loss, most of which failed to display choroidal vessels as en-face OCT images did. Representative OCTA images, en-face OCT images, binarized images and image comparison in a subject with SCT of $378 \mu \mathrm{m}$ are shown in Fig. 3.

The choroidal vascular display on OCTA images and image similarity between OCTA and en-face OCT images at fixed and percentile depths are shown in Table 2 and Table 3, respectively. The optimal depth in fixed depth series was $100 \mu \mathrm{m}$ beneath BM where all OCTA images displayed choroidal vessels appropriately and similarity between OCTA and en-face OCT images peaked at $82.0 \pm 2.6 \%$. The optimal depth in percentile depth series was $25.0 \%$ of SCT beneath BM where 81 out of 91 (89.0\%) OCTA images appropriately displayed choroidal vessels and similarity reached $79.6 \pm 3.8 \%$. In both series, OCTA images had limited capability in visualizing extremely deep choroidal vessels compared with en-face OCT images. Considering that the OCTA images at $25.0 \%$ of SCT beneath BM might not always display choroidal vessels and the minor variation in choroidal thickness measurement might result in a change of the slab localization, we selected $100 \mu \mathrm{m}$ beneath BM as the optimal depth for measuring deep CVD with OCTA.

\section{Choroidal vascular density and correlating factors in healthy subjects}

Among the 91 healthy eyes, the mean CVD at $100 \mu \mathrm{m}$ beneath $\mathrm{BM}$ was $56.5 \pm 10.9 \%$. Univariate analysis showed that CVD at $100 \mu \mathrm{m}$ beneath $\mathrm{BM}$ was significantly correlated with SCT $(P=0.003)$ but not with age $(P=0.40)$ or AL $(P=0.67)$. Multivariate linear regression revealed that for every increase in SCT of $100 \mu \mathrm{m}, \mathrm{CVD}$ at $100 \mu \mathrm{m}$ beneath BM increased by 3.9\% (95\% confidence interval 1.3 to $6.5 \%, P=0.004)$. The CVD at $100 \mu \mathrm{m}$ beneath $\mathrm{BM}$ had no significant correlation with age $(P=0.49)$ or $\mathrm{AL}(P=0.72)$.

\section{Choroidal vascular density in CSC subjects and comparison with controls}

In one-way ANOVA, the CVD at $100 \mu \mathrm{m}$ beneath BM was significantly increased in the 22 CSC eyes compared with 22 matched controls $(62.3 \pm 6.6 \%$ and $54.1 \pm 8.0 \%$, $P=0.001)$. In ANCOVA where SCT was adjusted as covariates, the CVD at $100 \mu \mathrm{m}$ beneath $\mathrm{BM}$ in CSC eyes was still significantly larger than that in controls $(P=$ 0.02). Representative OCTA images at $100 \mu \mathrm{m}$ beneath $\mathrm{BM}$ in CSC and healthy eyes are shown in Fig. 4.

\section{Discussion}

By systematically evaluating deep choroidal OCTA images in healthy and CSC eyes, the current study not only suggested the capability of SS-OCTA in displaying choroidal vessels, but also identified CVD at $100 \mu \mathrm{m}$ beneath $\mathrm{BM}$ as a useful OCTA parameter. This parameter was stable across various age and axial length groups, and was valuable in reflecting choroidal vascular status.

There had been an impression that OCTA might not be suitable for imaging the choroid beneath choriocapillaris. According to Diaz et al., motion contrast signals produced bright vessel outlines only in areas with overlying RPE atrophy, and dark signals beneath intact RPE was deemed as absence of blood flow [8]. According to our study, within a range of choroidal depths, dark signals in OCTA images corresponded to vessel lumen in OCT B-scan, and deep choroidal vessels manifested as dark stripes in OCTA images. This phenomenon could be related with several underlying mechanisms including fringe washout effect, signal attenuation, and threshold masking. Fringe washout is the signal loss due to high blood flow velocity exceeding system imaging speed [16-18]. Attenuation is the signal blockage by RPE which reduces a significant portion of light entering choroid [13, 19]. Threshold masking is a process of signal removal in OCTA image building which ensures that only valid motion contrast signals were included and potential noises were excluded [19]. In our study, OCTA images and en-face OCT images displayed choroidal vessels as similar dark stripes within a range of choroidal depths. Therefore, the notion that OCTA cannot be used for deep choroid imaging might be biased and incomprehensive. 


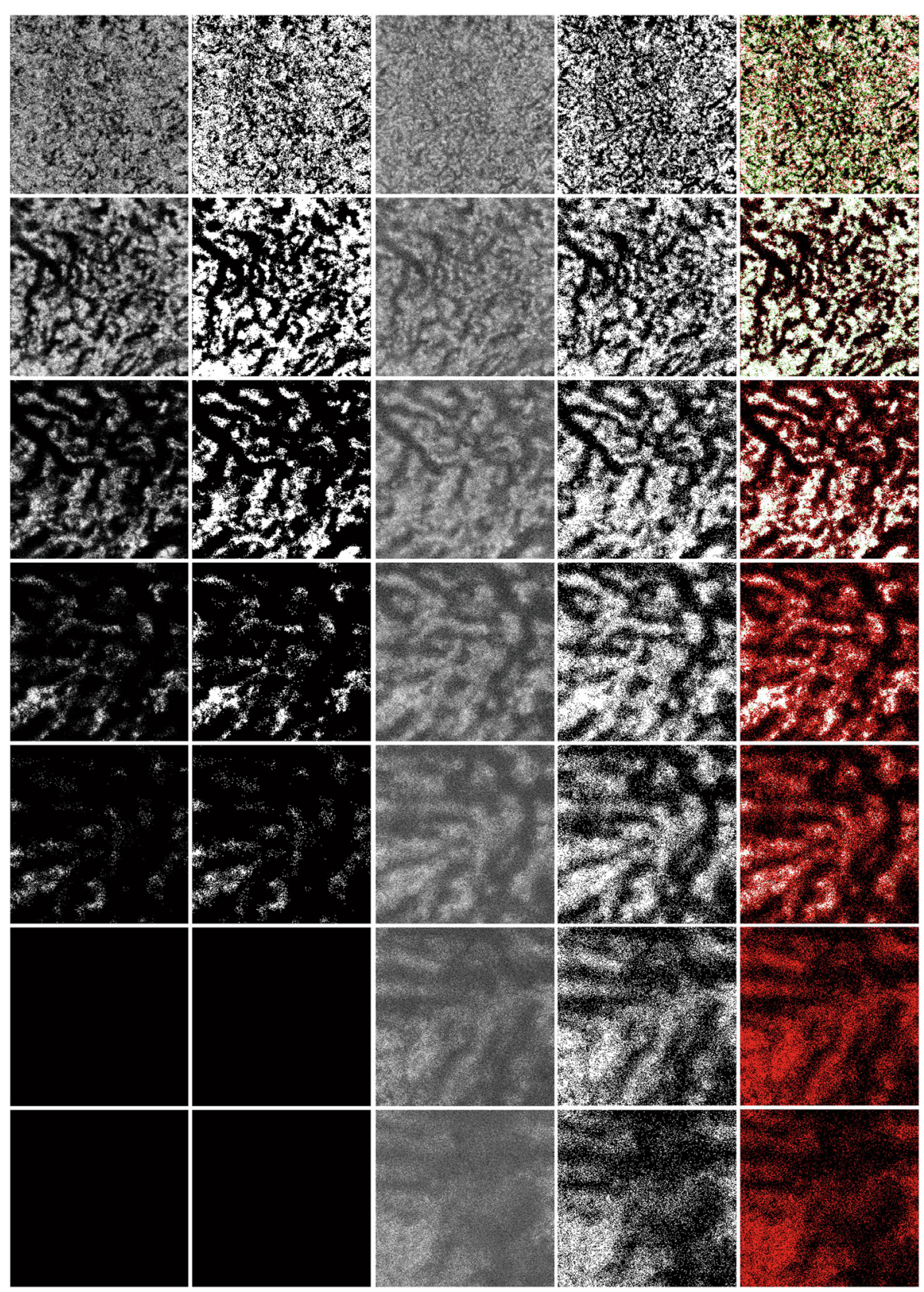

Fig. 3 Representative OCTA and en-face OCT images at various choroidal depths. The SS-OCTA scan was performed in the right eye of a healthy subject in his 6th decade of life with central choroidal thickness of $378 \mu \mathrm{m}$. The five columns, from left to right, showed original OCTA images, binarized OCTA images, original en-face OCT images, binarized en-face OCT images, and comparison images. In comparison image, a pixel would be shown in white, black, green, and red, respectively, when the corresponding pixel was white in both OCTA and en-face OCT, black in both OCTA and en-face OCT, white in OCTA but black in en-face OCT, and black in OCTA but white in en-face OCT. The seven rows, from top to bottom, showed images at $50 \mu \mathrm{m}, 100 \mu \mathrm{m}, 150 \mu \mathrm{m}, 200 \mu \mathrm{m}, 250 \mu \mathrm{m}, 300 \mu \mathrm{m}$, and $350 \mu \mathrm{m}$ beneath Bruch membrane (BM). At $100 \mu \mathrm{m}$ beneath BM, OCTA image displayed choroidal vessels as clearly as en-face OCT image, and the similarity between OCTA and its en-face OCT counterpart peaked at $82.0 \%$

We identified $100 \mu \mathrm{m}$ beneath $\mathrm{BM}$ as the optimal depth for the current SS-OCTA device in depicting choroidal vessels for two reasons. First, in all healthy subjects, OCTA images at $100 \mu \mathrm{m}$ beneath BM were able to display choroidal vessels appropriately. Second,
OCTA and en-face OCT images had greatest similarity at $100 \mu \mathrm{m}$ beneath $\mathrm{BM}$ in almost all eyes with various choroidal thickness. The above facts suggested that capability of SS-OCTA in depicting choroidal vessels was likely to be determined by the intrinsic penetration of 
Table 2 The Number of OCTA Images Displaying Choroidal Vessels and Similarity Between OCTA and en-face OCT Images in Fixed Depth Series

\begin{tabular}{lll}
\hline $\begin{array}{l}\text { Depth beneath BM } \\
(\mu \mathrm{m})\end{array}$ & $\begin{array}{l}\text { Number (\%) of OCTA images displaying choroidal } \\
\text { vessels }\end{array}$ & $\begin{array}{l}\text { Similarity between OCTA and en-face OCT images (mean } \pm \\
\text { SD\%) }\end{array}$ \\
\hline $50(n=91)$ & $27(29.7)$ & $74.7 \pm 4.2$ \\
$100(n=91)$ & $91(100.0)$ & $\mathbf{8 2 . 0} \pm \mathbf{2 . 6}$ \\
$150(n=89)$ & $79(88.8)$ & $76.7 \pm 6.5$ \\
$200(n=86)$ & $67(77.9)$ & $72.0 \pm 7.2$ \\
$250(n=69)$ & $35(51.5)$ & $66.5 \pm 6.2$ \\
$300(n=58)$ & $6(10.3)$ & $58.8 \pm 6.4$ \\
$350(n=44)$ & $0(0.0)$ & $57.4 \pm 7.2$ \\
$400(n=23)$ & $0(0.0)$ & $57.3 \pm 5.7$ \\
$450(n=7)$ & $0(0.0)$ & $52.9 \pm 1.5$ \\
$500(n=4)$ & $0(0.0)$ & $50.5 \pm 0.5$ \\
$550(n=1)$ & $0(0.0)$ & 50.0 \\
\hline
\end{tabular}

The optimal fixed depth with the largest percentage of OCTA images displaying choroidal vessels and the greatest similarity between OCTA and en-face OCT images is shown in bold

BM Bruch membrane, OCTA optical coherence tomography angiography, OCT optical coherence tomography

the SS-OCTA device, rather than the imaging preference of specific choroidal layers. Morphological studies from autopsy eyes showed that the thickness of BM and choriocapillaris ranged from $2.0 \mu \mathrm{m}$ to $4.7 \mu \mathrm{m}$ and from $6.5 \mu \mathrm{m}$ to $9.8 \mu \mathrm{m}$, respectively [20]. In vivo investigation showed that subfoveal thickness of Sattler's layer was $87 \pm 56 \mu \mathrm{m}$ [21]. We thereby inferred that the choroidal OCTA image at $100 \mu \mathrm{m}$ beneath BM was likely to describe vasculatures around the boundary between Sattler's and Haller's layer.

Because deep choroidal vessels were displayed as dark stripes in OCTA images, the quantification of vascular density should be conducted by manual calculation of the proportion of dark signals instead of the automatic measurement of area of bright pixels generated by the built-in software. Chan et al. reported their OCTA measurements of CVD in deep choroid of CSC patients before and after photodynamic therapy (PDT). They agreed that the dark stripes in deep choroidal OCTA images reflected blood vessels. However, they measured CVD with a built-in algorithm which calculated the proportion of bright signals, normally used for measuring choriocapillary blood flow [9]. They reported a reduction in choroidal vessel diameter but an increase in vascular density after PDT, which was self-contradictory and inconsistent with a number of previous observations on PDT treatment $[22,23]$.

We revealed that CVD measured by SS-OCTA at $100 \mu \mathrm{m}$ beneath BM is a useful parameter for quantifying choroidal vascular status. First, no manual calibration of SCT is needed in calculating CVD. The fixed depth, or $100 \mu \mathrm{m}$ beneath $\mathrm{BM}$, for image generation is likely to minimize potential measurement bias among different technicians. Second, this parameter was correlated with choroidal thickness and was relatively stable across various age and refraction groups. One previous study by Fujiwara et al. [3] utilized en-face OCT for the measurement of CVD at 50\% SCT beneath BM and

Table 3 The Number of OCTA Images Displaying Choroidal Vessels and Similarity Between OCTA and en-face OCT Images in Percentile Depth Series

\begin{tabular}{lll}
\hline $\begin{array}{l}\text { Depth beneath BM } \\
(\% \mathrm{SCT})\end{array}$ & $\begin{array}{l}\text { Number (\%) of OCTA images displaying choroidal } \\
\text { vessels }\end{array}$ & $\begin{array}{l}\text { Similarity between OCTA and en-face OCT images (mean } \pm \\
\text { SD\%) }\end{array}$ \\
\hline $12.5(n=91)$ & $25(27.5)$ & $71.9 \pm 6.5$ \\
$\mathbf{2 5 . 0}(\boldsymbol{n}=\mathbf{9 1})$ & $\mathbf{8 1 ( 8 9 . 0 )}$ & $\mathbf{7 9 . 6} \pm \mathbf{3 . 8}$ \\
$37.5(n=91)$ & $80(87.9)$ & $77.9 \pm 5.4$ \\
$50.0(n=91)$ & $78(85.7)$ & $73.6 \pm 8.3$ \\
$62.5(n=91)$ & $48(52.8)$ & $71.6 \pm 9.2$ \\
$75.0(n=91)$ & $38(41.8)$ & $69.2 \pm 10.6$ \\
$87.5(n=91)$ & $26(28.6)$ & $65.8 \pm 12.0$ \\
\hline
\end{tabular}

The optimal percentile depth with the largest percentage of OCTA images displaying choroidal vessels and the greatest similarity between OCTA and en-face OCT images is shown in bold

BM Bruch membrane, SCT central choroidal thickness, OCTA optical coherence tomography angiography, OCT optical coherence tomography 


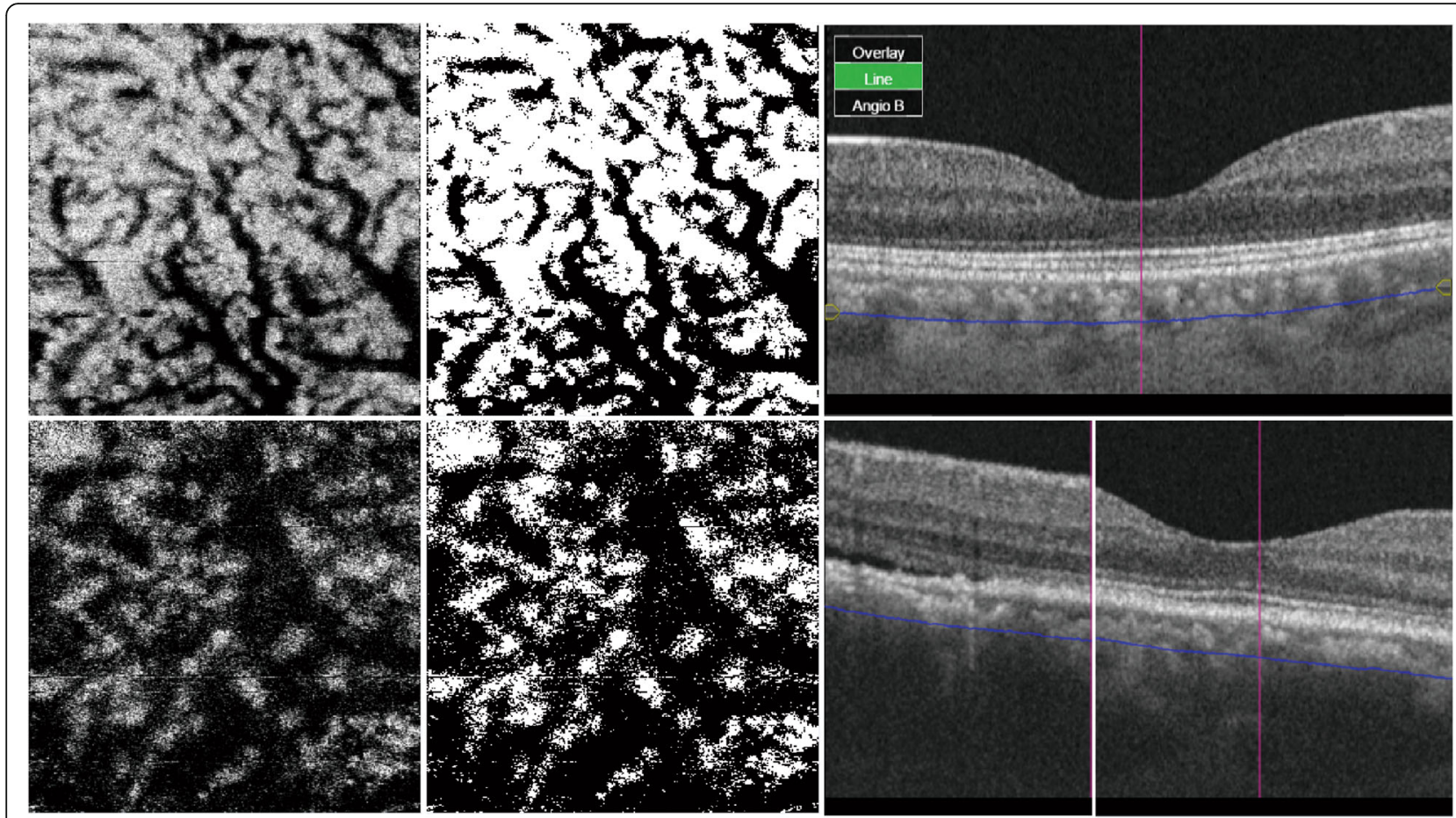

Fig. 4 Representative OCTA images at $100 \mu \mathrm{m}$ beneath Bruch membrane (BM) in healthy and central serous chorioretinopathy (CSC) eyes. (Top left) OCTA image of the right eye of a 31-year-old healthy female whose subfoveal choroidal thickness (SCT) was $288 \mu \mathrm{m}$ and axial length (AL) was $23.40 \mathrm{~mm}$. (Top middle) The binarized OCTA image of the healthy eye. (Top right) B-scan of the healthy eye with the arc line showing the imaging depth. (Bottom left) OCTA image of the right eye of a 41-year-old CSC male whose SCT was $507 \mu \mathrm{m}$ and AL was $23.05 \mathrm{~mm}$. (Bottom middle) The binarized OCTA image of the CSC eye. (Bottom right) OCT B-scan of the CSC eye with dilated choroidal vessels and subretinal fluid. Based on the OCTA image at $100 \mu \mathrm{m}$ beneath BM, the choroidal vascular density was 58.2 and $71.5 \%$ in the healthy eye and the CSC eye, respectively

reported that CVD was significantly correlated with SCT but not with AL, which is similar to our findings. However, they reported that CVD measured with en-face OCT was negatively correlated with age, which might be due to relatively large sample size and broad sample age. Third, the value of this parameter in reflecting choroidal vascularity was also tested and verified in a series of CSC eyes. A plenty of studies have shown increased choroidal blood flow or dilated choroidal vessels in CSC patients $[12,24]$. In our study, CVD measured by SSOCTA at $100 \mu \mathrm{m}$ beneath BM was increased in CSC eyes, consistent with previous findings. One may argue that the increased CVD value in CSC eyes might be related with thick choroid. To eliminate the potential influence from SCT, we compared CVD between groups after adjusting for SCT and the difference remained significant. One may also argue that the presence of subretinal fluid or pigment epithelium detachment may produce shadowing artifacts in OCTA images of underlying structures and may result in an overestimation of CVD in deep choroid. However, the round dark "shadow" produced by subretinal fluid was almost invisible in deep choroid, because there was mild, universal, and uniform signal loss in deep choroid. Based on the above considerations, we believe that CVD at $100 \mu \mathrm{m}$ beneath BM measured by OCTA is a potentially valuable and convenient parameter in future studies.

Despite the potential value of SS-OCTA in visualizing choroidal vessels and measuring CVD at $100 \mu \mathrm{m}$ beneath $\mathrm{BM}$, we have to admit that the capability of SS-OCTA in visualizing deep choroidal vasculatures is limited in extremely deep choroid where signal attenuation is significant. Signal attenuation in OCTA images results in more dark signals than actual and thereby causes overestimation of CVD [11, 18].

There were several limitations in the current study. First, the healthy group included more females than males, although no significant difference of CVD was observed between males and females $(P=0.36)$. Second, the healthy group included only 91 subjects. The correlation between CVD and SCT as well as the stability of CVD among various age and refraction groups need further confirmation in a larger sample. Third, our result is only applicable to the current SS-OCTA device, and the capability of other OCTA devices in visualizing deep choroid and measuring CVD remains unknown. Fourth, we observed choroidal OCTA images at predetermined depths with $50 \mu \mathrm{m}$ intervals instead of a consecutive 
series of depths. Studies with refined choroidal depth intervals might add to our current knowledge in SSOCTA imaging of deep choroid. Fifth, choroidal imaging was limited in a $3.0 \times 3.0 \mathrm{~mm}$ macular area. A broader imaging coverage might be needed in future studies.

\section{Conclusions}

The current SS-OCTA device was useful in visualizing choroidal vasculatures within a range of choroidal depths. Notably, this SS-OCTA device was able to measure CVD at $100 \mu \mathrm{m}$ beneath BM, which was a useful parameter in quantifying choroidal vascular status. The study improved our understanding in OCTA technique and expanded its application in deep choroid imaging.

\section{Abbreviations}

OCT: Optical coherence tomography; SS-OCT: Swept source optical coherence tomography; OCTA: Optical coherence tomography angiography; SS-OCTA: Swept source optical coherence tomography angiography; CVD: Choroidal vascular density; SCT: Subfoveal choroidal thickness; CSC: Central serous chorioretinopathy; RPE: Retinal pigment epithelium; BCVA: Best corrected visual acuity; IOP: Intraocular eye pressure; AL: Axial length; BM: Bruch membrane

\section{Acknowledgements}

The authors acknowledge Dr. Yanlong Li for his statistical support.

\section{Authors' contributions}

EW and $Y C$ designed the study. EW, $X Z$, and JY collected and analyzed all data. EW, $X Z, J Y, Y C$ interpreted the data and results. EW drafted the manuscript. $X Z, J Y$, and $Y C$ reviewed the manuscript. All authors read and approved the final manuscript.

\section{Authors' information}

Null.

\section{Funding}

The study was supported by the Non-Profit Central Research Institute Fund of Chinese Academy of Medical Sciences (Grant No. 2018PT32029). The funding organization had no role in the design or conduct of this research.

\section{Availability of data and materials}

The datasets used and/or analysed during the current study are available from the corresponding author on reasonable request.

\section{Ethics approval and consent to participate}

The study was in compliance with the tenets of the Declaration of Helsinki. The Ethics Committee of Peking Union Medical College Hospital approved the study protocol. All participants gave written informed consent

\section{Consent for publication}

Not applicable.

\section{Competing interests}

The authors declare that they have no competing interests.

Received: 18 March 2020 Accepted: 29 July 2020

Published online: 05 August 2020

\section{References}

1. Motaghiannezam R, Schwartz DM, Fraser SE. In vivo human choroidal vascular pattern visualization using high-speed swept-source optical coherence tomography at $1060 \mathrm{~nm}$. Invest Ophthalmol Vis Sci. 2012;53: 2337-48.

2. Adhi M, Ferrara D, Mullins RF, et al. Characterization of choroidal layers in normal aging eyes using enface swept-source optical coherence tomography. PLoS One. 2015;10:e0133080.
3. Fujiwara A, Morizane $Y$, Hosokawa M, et al. Factors affecting Choroidal vascular density in Normal eyes: quantification using en face swept-source optical coherence tomography. Am J Ophthalmol. 2016;170:1-9.

4. Wang JC, Lains I, Providencia J, et al. Diabetic Choroidopathy: Choroidal vascular density and volume in diabetic retinopathy with swept-source optical coherence tomography. Am J Ophthalmol. 2017;184:75-83.

5. Baek J, Lee JH, Jung BJ, et al. Morphologic features of large choroidal vessel layer: age-related macular degeneration, polypoidal choroidal vasculopathy, and central serous chorioretinopathy. Graefes Arch Clin Exp Ophthalmol. 2018;256:2309-17.

6. Ang M, Tan ACS, Cheung CMG, et al. Optical coherence tomography angiography: a review of current and future clinical applications. Graefes Arch Clin Exp Ophthalmol. 2018;256:237-45

7. Sambhav K, Grover S, Chalam KV. The application of optical coherence tomography angiography in retinal diseases. Surv Ophthalmol. 2017:62:838-66.

8. Diaz JD, Wang JC, Oellers P, et al. Imaging the deep Choroidal vasculature using spectral domain and swept source optical coherence tomography angiography. J Vitreoretin Dis. 2018;2:146-54.

9. Chan SY, Pan CT, Wang Q, et al. Optical coherent tomographic angiographic pattern of the deep choroidal layer and choriocapillaris after photodynamic therapy for central serous chorioretinopathy. Graefes Arch Clin Exp Ophthalmol. 2019:257:1365-72.

10. Choi W, Mohler KJ, Potsaid B, et al. Choriocapillaris and choroidal microvasculature imaging with ultrahigh speed OCT angiography. PLoS One. 2013;8:e81499.

11. Wang JC, Laíns I, Silverman RF, et al. Visualization of choriocapillaris and choroidal vasculature in healthy eyes with en face swept-source optical coherence tomography versus angiography. Trans VisSci Tech. 2018;7:25.

12. Kuroda Y, Ooto S, Yamashiro K, et al. Increased choroidal vascularity in central serous chorioretinopathy quantified using swept-source optical coherence tomography. Am J Ophthalmol. 2016;169:199-207.

13. Chen FK, Viljoen RD, Bukowska DM. Classification of image artefacts in optical coherence tomography angiography of the choroid in macular diseases. Clin Exp Ophthalmol. 2016;44:388-99.

14. Ghasemi Falavarjani K, Al-Sheikh M, Akil H, Sadda SR. Image artefacts in swept-source optical coherence tomography angiography. Br J Ophthalmol. 2017;101:564-8.

15. Otsu N. A threshold selection method from gray-level histograms. IEEE Trans Syst, Man, Cybern. 1979:9:62-6.

16. Jia Y, Bailey ST, Hwang TS, et al. Quantitative optical coherence tomography angiography of vascular abnormalities in the living human eye. Proc Natl Acad Sci U S A. 2015:112:E2395-402.

17. Hendargo HC, McNabb RP, Dhalla AH, et al. Doppler velocity detection limitations in spectrometer-based versus swept-source optical coherence tomography. Biomed Opt Express. 2011;2:2175-88.

18. Hua $\mathrm{R}$, Wang $\mathrm{H}$. Dark signals in the choroidal vasculature on optical coherence tomography angiography: an artefact or not? J Ophthalmol. 2017:2017:5498125.

19. Spaide RF, Fujimoto JG, Waheed NK. Image artifacts in optical coherence tomography angiography. Retina. 2015:35:2163-80.

20. Ramrattan RS, van der Schaft TL, Mooy CM, et al. Morphometric analysis of Bruch's membrane, the choriocapillaris, and the choroid in aging. Invest Ophthalmol Vis Sci. 1994;35:2857-64.

21. Esmaeelpour M, Kajic V, Zabihian B, et al. Choroidal Haller's and Sattler's layer thickness measurement using 3-dimensional 1060-nm optical coherence tomography. PLoS One. 2014;9:e99690.

22. Demirel S, Ozcan G, Yanik O, et al. Vascular and structural alterations of the choroid evaluated by optical coherence tomography angiography and optical coherence tomography after half-fluence photodynamic therapy in chronic central serous chorioretinopathy. Graefes Arch Clin Exp Ophthalmol. 2019;257:905-12.

23. Izumi T, Koizumi H, Maruko I, et al. Structural analyses of choroid after halfdose verteporfin photodynamic therapy for central serous chorioretinopathy. Br J Ophthalmol. 2017;101:433-7.

24. Tang PH, Shields R, Silva RA. Optical coherence tomography angiography findings in chronic central serous Chorioretinopathy after photodynamic therapy. Ophthalmic Surg Lasers Imaging Retina. 2019;50:25-32.

\section{Publisher's Note}

Springer Nature remains neutral with regard to jurisdictional claims in published maps and institutional affiliations. 